\title{
INVESTIGACIÓN EDUCATIVA
}

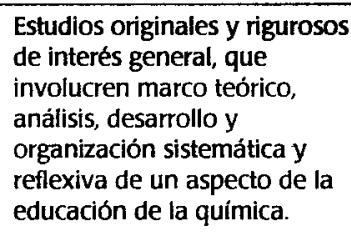

\section{Construcción del concepto de reacción química*}

\author{
Caruso, M. F., Castro, M., ${ }^{1}$ Domínguez Castiñeiras, J., ${ }^{2}$ \\ García-Rodeja, E., ${ }^{2}$ Iturralde, C., ${ }^{7}$ Rocha, A., ${ }^{7}$ y Scandroli, $N .{ }^{1}$
}

\begin{abstract}
Is a claim of the first part of this work to investigate what kind of interpretation is possible to obtain from students, which are driving themselves to the end of the secondary school, when a microscopic representation of a chemical reaction is proposed. The qualitative data are obtained through a paper-and-pencil test specially drawn from two groups of students between 16 and 17 years old.

In the second part, the treatment of the chemical reaction concept is explored in some common textbooks used at school. The analysis show a correlation with the student's answers obtained in the first part of this work.
\end{abstract}

\section{Introducción}

El interés por el concepto de reacción química que construyen los alumnos hasta llegar a la Universidad, radica en el hecho de que se considera fundamental para interpretar el de equilibrio químico, tal como este último se plantea en muchos de los textos de Química General utilizados en los primeros cursos universitarios. Sería importante en este sentido, que los alumnos interpretasen una reacción química utilizando el modelo de partículas y esencialmente la concibiesen como un reacomodamiento, una recombinación de los átomos, que incluya además la idea de que se trata de un sistema dinámico (lo cual involucra el aspecto energético de las reacciones químicas).

Muchos son los estudios que se han realizado sobre cómo interpretan los alumnos reacciones químicas de la vida cotidiana tales como combustión y oxidación de metales Driver, (1989). Tanto es así que han llegado a desarrollarse clasificaciones de las interpretaciones de los alumnos, una

\footnotetext{
- El presente trabajo surge paralelamente al desarrollo de las investigaciones que sobre la adquisición del concepto de Equilibrio Químico se realizan en el Grupo de Didáctica de las Ciencias Experimentales de la Facultad de Ingenieria de la UNCPBA (Argentina) con el asesoramiento del grupo homólogo de la Universidad de Santiago de Compostela (España).

1 Departamento de Profesorado. Facultad de Ingeniería. UNCPBA. Av. del Valle 5737 (7400) Olavarría.

2 Departamento de Didáctica de las Ciencias Experimentales. Universidad de Santiago de Compostela (España)

Recibido: 29 de octubre de 1997; Aceptado: 12 de marzo de 1998.
}

de las cuales, la de Anderson (1986) considera que existen cinco categorías de interpretación utilizadas por los alumnos para el concepto de reacción química. En un extremo de esta clasificación estarían las respuestas que consideran, a nivel macroscópico, que hay reacción química cuando cambian algunas de las propiedades de las sustancias y a nivel microscópico, que varían la forma, el tamaño y el color de las partículas, asignándoles las características macroscópicas de las sustancias (Chastrette, 1991). En el extremo opuesto, podemos considerar aquellas respuestas que tienen en cuenta, a nivel micro, la conservación de la identidad atómica, la recombinación de los átomos y reconocen la formación de nuevas sustancias.

La primera parte de este trabajo tiene por objeto indagar cómo interpretan los alumnos que se hallan hacia el final de la escuela secundaria, una reacción química, propuesta mediante una "representación" de partículas. El instrumento de investigación utilizado consiste en representaciones de partículas y fue especialmente diseñado para que las explicaciones se basasen en el modelo microscópico de materia, ya que se ha podido comprobar que los alumnos no usan las explicaciones a nivel microscópico si no se los induce a hacerlo (Chastrette, 1991). En realidad se pretende saber si el alumno posee una idea de reacción química como reacomodamiento; como recombinación de átomos. Esta forma de obtener información no permite indagar directamente acerca del aspecto dinámico de una reacción química, esto constituye el paso siguiente en la investigación.

Las ideas detectadas en este trabajo coinciden con los resultados de otras investigaciones (Anderson, 1986; Nakhleh, 1992), pero llama la atención una respuesta muy generalizada entre los alumnos de los dos grupos objeto de estudio y es que "sólo hay reacción química si se unen dos sustancias distintas". Esto podría estar reflejando una fuerte incidencia de la idea de "ecuación química" sobre la idea de reacción química (Caruso, 1994), lo cual puede atribuirse en parte a la forma en que tanto en el aula, como en la bibliografía química más usada en la escuela secundaria, se trata el tema de las reacciones químicas.

En la segunda parte de este trabajo se hace un análisis de la bibliografía más usada en Química, a nivel secundario, que pretende mostrar la evolución del concepto de reacción química a lo largo de dichos textos y paralelamente, el uso 
del modelo de partículas, particularmente en relación con este concepto.

\section{DESARROLLO DE LA INVESTIGACIÓN}

\section{PRIMERA PARTE}

Muestra: Se trabaja con alumnos de dos cursos de $4^{\circ}$ año, de un mismo docente, de escuela secundaria. Los cursos seleccionados poseen características bien diferenciadas en cuanto a su especialización y por ende probablemente en cuanto a su inclinación hacia la ciencia. La edad de los alumnos oscila entre 16 y 17 años.

Ambos grupos pertenecen a la Escuela Comercial de la ciudad de Tandil (Argentina). Esta escuela posee, además de la orientación contable, dos orientaciones más, la científica y la pedagógica. Existen importantes diferencias entre ambas en cuanto al perfil, lo cual se traduce, en una primera instancia, en la amplitud y profundidad con que se trabaja el contenido científico.

- Grupo A: $4^{\circ}$ año, turno mañana. Número de alumnos: 22 . Orientación científica.

- Grupo B: $4^{\circ}$ año, turno tarde. Número de alumnos: 28 . Orientación pedagógica.

Instrumento de recolección de datos: consiste en una prueba de lápiz y papel con varios ítems. Cada ítem es un dibujo que representa, mediante partículas (átomos), el contenido de un recipiente en dos momentos distintos (figura 1). a)

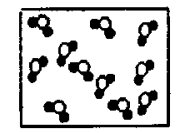

b)

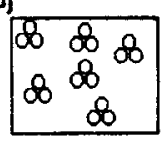

c)

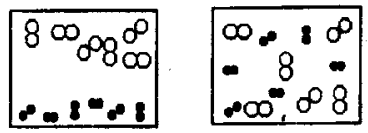

d]

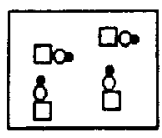

e)

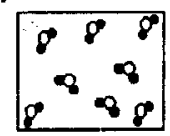

ก

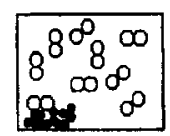

g)

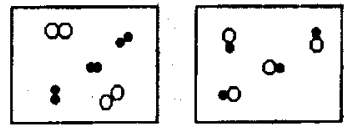

Figura 1. ¿En cuáles de los siguientes casos se está representando una reacción quimica? Los símbolos $\bullet, \square$ y $\bigcirc$ representan partículas.
Se pide que el alumno decida en cada caso si ha ocurrido o no una reacción química y justifique su decisión.

Cabe destacar que en el momento de la prueba se aclara a cada grupo de alumnos que las representaciones planteadas en cada ítem corresponden al contenido de un recipiente en dos momentos diferentes y se insiste en la importancia de justificar para cada caso si pudo haber ocurrido o no una reacción quimica.

La forma en que se ha planteado la prueba, pretende inducir al alumno a utilizar explicaciones microscópicas en todos los items, ya que son muchos los trabajos que muestran que los alumnos no usan espontáneamente el modelo de partículas en sus explicaciones, es necesario inducirlos a ello (Chastrette, 1991).

\section{Resultados obtenidos y análisis cuantitativo}

En la tabla 1 aparecen los porcentajes de respuestas correctas y alternativas para cada uno de los items. Se consideran respuestas correctas aquellas que deciden acertadamente en cada caso si se trata o no de una reacción química y lo justifican. Se clasifican como NC ("No Contesta") los ítems no resueltos o las respuestas no justificadas.

Tabla 1.

\begin{tabular}{|c|c|c|c|c|c|c|c|}
\hline \multicolumn{4}{|c|}{ GRUPO A $(n=22)$} & \multicolumn{4}{|c|}{ GRUPO B $(n=28)$} \\
\hline Item & $\begin{array}{c}\text { Correcto } \\
\%\end{array}$ & $\begin{array}{l}\text { Alterna- } \\
\text { tivo \% }\end{array}$ & $\begin{array}{l}N C \\
\%\end{array}$ & Hem & $\begin{array}{c}\text { Correcto } \\
\%\end{array}$ & $\begin{array}{c}\text { Alterna- } \\
\text { tivo \% }\end{array}$ & $\begin{array}{l}\text { NC } \\
\%\end{array}$ \\
\hline $\mathbf{a}$ & 81 & 14 & 5 & $\mathbf{a}$ & 32 & 39 & 29 \\
\hline b & 73 & 27 & - & b & 54 & 25 & 21 \\
\hline c & 72 & 5 & 23 & G & 46 & 18 & 36 \\
\hline$d$ & 77 & 14 & 9 & $d$ & 35 & 29 & 36 \\
\hline et & 73 & 18 & 9 & 4 & 39 & 22 & 39 \\
\hline $\mathbf{f}$ & 50 & 18 & 32 & $f$ & 54 & 18 & $\mathbf{2 8}$ \\
\hline $\mathbf{g}$ & 45 & 45 & 10 & $\mathbf{g}$ & - & 54 & 46 \\
\hline
\end{tabular}

Analizando los ítems (a) a (e), los porcentajes de respuestas correctas para el grupo A son mayores que para el grupo B. El grupo A mantiene porcentajes similares de respuestas correctas para todos los items (entre 72 y $81 \%$ ), lo cual podría estar indicando que los alumnos poseen una idea de reacción química que pueden aplicar a los distintos casos, mientras que el grupo $B$ presenta resultados más dispersos (entre 32 y el $54 \%$ ) que estarían indicando una dependencia de la respuesta con el ejemplo particular que se esté considerando. No parece existir, entre estos alumnos, un concepto de reacción química aplicable en cualquier situación. 
Es notable, por ejemplo, que en el ítem (a), se da el mayor número de respuestas correctas para el grupo A y el menor para el grupo $\mathbf{B}$.

En cuanto al ítem (f), los resultados son muy semejantes para ambos grupos pero es notable el elevado porcentaje de NC correspondiente al grupo A. Esto nos lleva a analizar el tipo de justificaciones que aparecen en las respuestas que hemos considerado alternativas. Todas las explicaciones son del tipo: "no hay reacción química porque sobran átomos". Esta dificultad adicional que aparece en la representación correspondiente a este ítem y que luego se repite de manera similar para el caso planteado en (g), complica el análisis cuantitativo.

La situación planteada en el caso (g), de no conservación de los átomos, es detectada sólo por unos pocos alumnos en el grupo A, siendo las justificaciones clasificadas como correctas para este ítem todas del tipo: "no es reacción química porque no se conservan los elementos". Esta cuestión no se incluye en los gráficos del apartado siguiente.

\section{Análisis cualitativo de las respuestas correctas}

Las respuestas correctas fueron clasificadas en respuestas con explicaciones macroscópicas y respuestas con explicaciones microscópicas. Cabe aclarar que hemos denominado explicaciones macroscópicas a aquellas que incluyen una justificación que no menciona el reacomodamiento de los átomos para explicar que exista reacción química. Por ejemplo: "El ozono se transforma en oxígeno" (para el ítem b).

No obstante, la interpretación de lo planteado en los items de la prueba, implica que el alumno ha hecho una representación microscópica de la situación. Esto es, su explicación no puede considerarse completamente macro.

Las figuras 2 y 3 muestran las respuestas correctas obtenidas separadas en macroscópicas y microscópicas para los grupos A y B respectivamente.

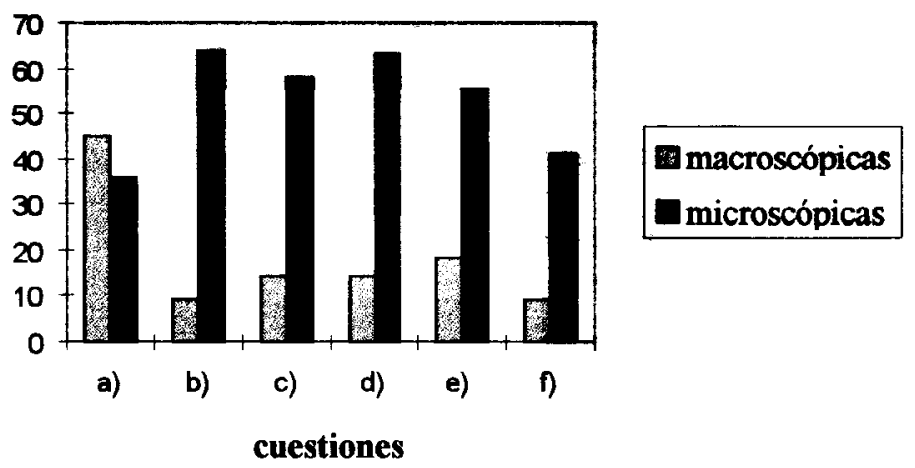

Figura 2. Porcentaje de respuestas correctas, separadas en macroscópicas y microscópicas para el grupo $A$.
De ambos gráficos se desprende que existe cierta similitud, desde el punto de vista cualitativo, en los resultados obtenidos para cada ítem por cada uno de los grupos. Para todos los ítems, excepto el a), en los dos grupos predominan las justificaciones microscópicas, si bien el grupo B utiliza menos el modelo de partículas en sus explicaciones.

Las justificaciones que hemos denominado "macroscópicas" surgen en mayor porcentaje, a pesar de que la prueba plantea explícitamente representaciones microscópicas, en los casos en que resulta posible relacionar lo propuesto con un "ejemplo" conocido por el alumno, tal es el caso del ítem (a). Así como está planteada esta cuestión, la representación resulta familiar para el alumno que reconoce que se trata de moléculas de agua que sufren un "cambio de estado".

\section{Análisis cualitativo de algunas respuestas alternativas}

Se apunta aquí a detectar las dificultades más sobresalientes en cuanto a la interpretación microscópica de las reacciones químicas. Para ello se transcriben a continuación algunas de las justificaciones más representativas.

Ítem (b):

"No es reacción química porque sólo se separan y juntan átomos iguales".

"No es reacción química porque no se producen relaciones con otros átomos".

Parecería existir la concepción de que "para que exista reacción química deben unirse átomos de distinta clase". Esta idea surge nuevamente cuando, entre las respuestas alternativas al ítem (g) (que no consideran la desaparición de dos átomos), aparecen un elevado número de justificaciones que consideran que existe reacción química porque: "se juntan átomos distintos".

De los resultados obtenidos podemos concluir: en dos grupos de alumnos en los que aparentemente los resultados desde el punto de vista cuantitativo resultan diferentes,

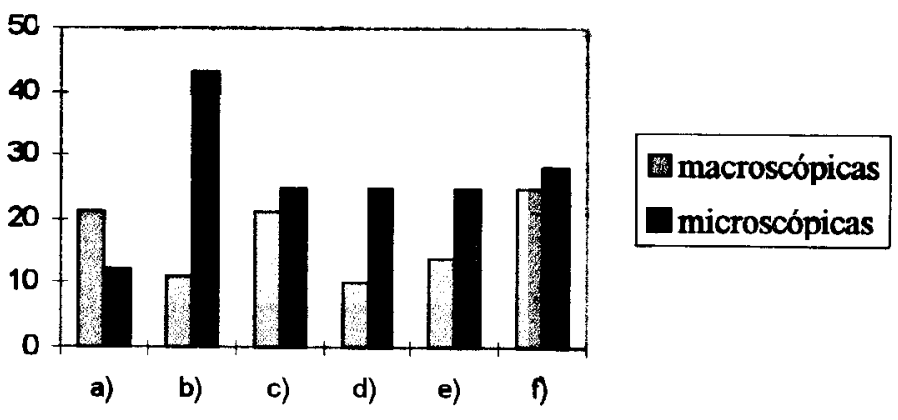

cuestiones

Figura 3. Porcentaje de respuestas correctas, separadas en macroscópicas y microscópicas para el Grupo B. 
aparecen concepciones semejantes a la hora de hacer explícita la idea de reacción química que poseen.

En ambos grupos, parece que la representación macroscópica se activa antes, ya que en el modelo interpretativo del alumno ocupa un status superior que la representación microscópica.

La distinción entre respuestas macroscópicas y microscópicas resulta interesante porque permite mostrar el hecho de que los alumnos se "apoyan" en las propiedades de las sustancias para reforzar la idea de si existe o no reacción química.

La idea alternativa detectada, según la cual, "hay reacción química si se unen dos sustancias distintas" o, a nivel microscópico, "ocurre una reacción química cuando se unen átomos distintos", y el predominio, entre las justificaciones, de aquellas que incluyen ejemplos de ecuaciones que muestran el cambio ocurrido, estarian reflejando una fuerte incidencia de la idea de "ecuación química" $(A+B \rightarrow C)$ sobre la de reacción química, que ya habíamos propuesto en un trabajo anterior (Caruso, 1994).

\section{SEGUNDA PARTE}

Analizamos aquí la forma de presentación del concepto de reacción química en los libros de texto utilizados por los alumnos involucrados en este estudio, como así también del modelo de materia que proponen. El objetivo es analizar si existe relación entre los resultados obtenidos en la primera parte del trabajo y la forma en que la bibliografía química (libros de texto) más usada en la escuela secundaria trata el tema.

\section{Análisis de textos}

Se limita a trabajar sobre tres de los textos de Química más usados a nivel secundario en los últimos años.

Para llevar a cabo el análisis de los libros de texto se utilizó el siguiente esquema:

1. ¿Dónde aparece tratado por primera vez el modelo corpuscular de la materia y cómo se lo introduce?

2. ¿Se aplica el modelo de partículas para la explicación de otros fenómenos a lo largo del texto?

3. ¿En qué capítulo del libro aparece por primera vez el concepto de reacción química y cómo se lo introduce?

4. ¿Cómo evoluciona el concepto de reacción química a lo largo del texto?

\section{TEXTO 1.}

QUIIMICA. General e Inorgánica. 1a Parte. Fernández Serventi, (1994), 29 edición, El Ateneo. Buenos Aires. Número total de capítulos: 35

1. Menciona en el primer capítulo que las moléculas se hallan en continuo movimiento. "Las fuerzas de atracción tienden a unirlas y las de repulsión a separarlas", según el estado físico de que se trate.

2. En el mismo capitulo, aparece explicado el concepto de evaporación con una breve descripción usando el "modelo de partículas" propuesto en este texto. En el capítulo 29 se usa este mismo modelo para explicar los conceptos de sólido molecular, sólido iónico, etcétera.

3. El concepto de reacción química aparece tratado por primera vez en el capítulo 4 donde se define como "el fenómeno por el que una o más sustancias se convierten en otras:

$$
\mathrm{A}+\mathrm{B} \rightarrow \mathrm{C}+\mathrm{D}+\mathrm{E} \text { (cambio permanente) }
$$

Se menciona la diferencia en la estructura molecular pero no se habla de ruptura y formación de enlaces. Se hace una interpretación con átomos. Aparece la idea de reacción reversible.

4. Se utilizan, a lo largo del texto, ecuaciones que representan reacciones de formación de los distintos tipos de compuestos, ácido-base, etcétera. Se trabaja en problemas de estequiometría con cálculos sobre ecuaciones químicas. Recién en el capítulo 27, en el tema Cinética Química y Equilibrio, reaparece la idea de reacción reversible y se presenta, como condición para que haya reacción química, el choque interatómico, interiónico o intermolecular.

\section{TEXTO 2.}

QUÍMICA GENERAL E INORGÁNICA. Biasioli, Weitz, Chandias (1981) 2a edición, Kapelusz, Buenos Aires. Número total de capítulos: 26

1. El modelo de materia discontinua aparece en el primer capítulo y se lo aplica a la explicación de los estados de la materia y sus cambios.

2. En el capítulo 6 retoma el modelo de partículas bajo el tema Teoría cinética de los gases, sin hacer relación alguna con lo tratado en el capítulo 1 . En el capítulo 16 utiliza el modelo para explicar el concepto de presión de vapor. En el capítulo 17 se trabaja la idea de cómo ocurren las reacciones químicas, utilizando la Teoría de las Colisiones.

3. La idea de reacción química aparece por primera vez en el capítulo 3 del libro y se plantea como aquel fenómeno por el que unas sustancias se transforman en otras. Inmediatamente después se presenta simbolizada a través de la ecuación química seguida de una interpretación molecular.

4. A lo largo del texto se maneja la idea inicial de que en una reacción química, unas sustancias se transforman en 
otras, acompañada de la ecuación correspondiente. Recién en el capítulo 17, de Cinética Química, se explica cómo se produce una reacción química, utilizando un modelo cinético-molecular y se menciona, por primera vez, la posibilidad de que existan reacciones químicas reversibles. En dicha explicación está implícito el hecho de que las partículas poseen energia, se mueven, chocan $y$ esto hace posible la ruptura y formación de enlaces nuevos.

\section{TEXTO 3.}

QUÍMICA. Aula Taller. J. M. Mautino (1992) 1a edición, Stella, Buenos Aires. Número total de capítulos: 8

1. Menciona el modelo de partículas de la materia en el primer capítulo y lo aplica a la explicación de los estados de la materia y de los cambios de estado. Predomina la idea de que "cuanto mayores sean las fuerzas de cohesión, menor resultará la distancia intermolecular y por lo tanto más restringido estará el movimiento de las partículas". El aspecto energético del modelo no está explícitamente trabajado.

2. Explica la presión de vapor en los gases (capítulo 7) y las Leyes de los Gases. También utiliza el modelo en la descripción de soluciones gas-gas y gas-líquido.

3. Hacia el final del primer capítulo se plantea la idea de

\section{Aprovechen la oferta! ivence en septiembre!}

El Consejo Editorial, después de decidir cambiar la periodicidad de trimestral a bimestral, ha abierto un periodo de oferta para captar más suscriptores.

Educación Química mantendrá el costo actual de suscripción hasta el mes de octubre de 1998. Ésta es la oportunidad para que aquellos cuya suscripción vence en 1998 nos envien $\$ 120.00$ o US\$25.00 (para los servicios al extranjero) y queden de esta manera suscritos durante 1999 a un precio subsidiado.

La oferta se extiende a quienes no están suscritos aún, y que podrán pagar su suscripción por los años de 1998 y 1999 con el pago de $\$ 200.00$ o US\$45.00

Hemos resuelto el problema de poder recibir pagos por la vía del cargo a una tarjeta de crédito nacional o internacional. Basta recortar y llenar el formato incluido en este ejemplar, y enviarlo firmado a nuestro apartado postal para renovar la suscripción por 1999 o hacer una de los años 1998 y 1999 (en este último caso los suscriptores recibirán en un solo envío todos los números de este año que ya han aparecido). Quienes deseen hacer el cargo a tarjeta de crédito pueden enviarnos el formato por fax al (525) 6223711 . reacción química como una transformación de la que resultan sustancias diferentes a las iniciales, con propiedades nuevas. Aporta ejemplos de distintos tipos de reacciones químicas y las diferencia delos fenómenos físicos sosteniendo que: fenómeno químico: cambio permanente; fenómeno físico: cambio no permanente.

4. A través de todo el texto, se identifica reacción química con ecuación química. En el capítulo 8 se usa la teoría de los choques para explicar cómo ocurre una reacción química. Recién al final del libro, se menciona la posibilidad de la existencia de reacciones reversibles.

\section{Conclusiones}

El modelo corpuscular de materia es expuesto por los libros de texto de Química, pero es muy poco usado en explicaciones.

El concepto de reacción química aparece en los primeros capítulos de los textos de Química, como aquel proceso por el cual, unas sustancias se transforman en otras, e inmediatamente se le asocia la idea de ecuación química, que es la que predomina en todo el texto. Hacia el final de los libros, en el tema Cinética Química se le da una interpretación molecular dinámica al concepto.

Los resultados obtenidos con la aplicación de la prueba a los alumnos concuerdan casi exactamente con la situación que hemos encontrado al analizar los textos que éstos utilizan en clases de Química.

Surgen de este trabajo, como implicaciones desde el punto de vista didáctico, la necesidad de insistir en clase, por un lado, en la diferenciación de los conceptos de reacción química y ecuación química y, por otro, en el uso de representaciones microscópicas de las reacciones químicas.

\section{Referencias bibliográficas}

Anderson, B., Pupil's explanations of some aspects of chemical reactions, Science Education, 70[5] 549-563, 1986.

Caruso, M.F., Castro, M. y Rocha, A., Memorias de la International Conference in Science and Mathematics Education. Towards approaches in the 21st. Century, Concepción, Chile, 1994.

Chastrette, M. y Franco, M., La reacción química: descripciones e interpretaciones de los alumnos de liceo, Ensenanza de las Ciencias, 9[3] 243-247, 1991

Driver, R., Guesne, E. y Tiberghien A., Ideas científicas en la infancia y la adolescencia, MEC: Morata. Madrid, 1989.

Nakhleh, M., Why some students don't learn chemistry? Chemical misconceptions, Journal of Chemical Education, 69[3], 191-196, 1992.

Pozo, J., Gómez Crespo, M., Limón, M. y Sanz Serrano, A., Procesos cognitivos en la comprensión de la ciencia: las ideas de los adolescentes sobre la química, CIDE Madrid, 1991. 